\title{
Identification and validation of colorectal neoplasia-specific methylation biomarkers based on CTCF-binding sites
}

\author{
Jiang Liu', ${ }^{1, *}$ Zhaoli Ding ${ }^{2,3, *}$, Guimei Li ${ }^{2,3, *}$, Li Tang1, Yu Xu4, Huayou Luo ${ }^{4}$, Jinhua \\ $\mathbf{Y i}^{4}$, Youwang Lu${ }^{4}$, Rui Mao5, Qiong Nan', Li Ren7, Tong Zhang ${ }^{4}$ and Kunhua Wang ${ }^{4}$ \\ ${ }^{1}$ Department of Reproduction and Genetics, The First Affiliated Hospital of Kunming Medical University, Kunming 650032, \\ Yunnan, China \\ ${ }^{2}$ Public Technical Service Center, Kunming Institute of Zoology, Chinese Academy of Sciences, Kunming 650032, Yunnan, \\ China \\ ${ }^{3}$ Kunming Biological Diversity Regional Center of Large Apparatus and Equipments, Chinese Academy of Sciences, Kunming \\ 650032, Yunnan, China \\ ${ }^{4}$ Department of Gastrointestinal Surgery, The First Affiliated Hospital of Kunming Medical University, Yunnan Institute of \\ Digestive Disease, Kunming 650032, Yunnan, China \\ ${ }^{5}$ School of Stomatology, Kunming Medical University, Kunming 650500, Yunnan, China \\ ${ }^{6}$ Department of Gastroenterology, The First Affiliated Hospital of Kunming Medical University, Yunnan Institute of Digestive \\ Disease, Kunming 650032, Yunnan, China \\ ${ }^{7}$ The First People's Hospital of Yunnan Province, Kunming 650031, Yunnan, China \\ "These authors have contributed equally to this work \\ Correspondence to: Kunhua Wang, email: kunhuawangProf@163.com \\ Keywords: colorectal cancer; early-detection; CTCF-binding site; DNA methylation; biomarker \\ Received: October 12, $2017 \quad$ Accepted: November 22, $2017 \quad$ Published: December 11, 2017 \\ Copyright: Liu et al. This is an open-access article distributed under the terms of the Creative Commons Attribution License 3.0 \\ (CC BY 3.0), which permits unrestricted use, distribution, and reproduction in any medium, provided the original author and source \\ are credited.
}

\section{ABSTRACT}

To date, the sensitivity of currently available biomarkers based on the methylation of gene promoters is suboptimal for detecting adenomas and earlystage colorectal cancer (CRC). We aimed to develop biomarkers with methylated DNA binding sites of the multifunctional transcriptional factor CTCF for early detection of CRC. Using combined analyses of genome-wide occupation and the methylation profile of CTCF-binding sites, we identified candidate CTCF-binding sites. Then, we applied methylation-sensitive high-resolution melting (MS-HRM) and mass spectrometry analysis to screen and validate these candidate sites in diverse sample sets. We identified a set of colorectal neoplasia-specific biomarkers with robust performance. The top five biomarkers were selected and recommended for early detection of colorectal neoplasia. All of the five novel biomarkers exhibited a more robust discriminatory performance than that by BMP3 and NDRG4, two currently acknowledged robust methylation biomarkers. When the five new biomarkers were considered as a marker panel and tumor-positive was defined as having two or more (of the five) positive biomarkers, the marker panel could achieve a sensitivity of $\mathbf{9 1 . 6 7 \%}$ for adenomas, $\mathbf{9 7 . 4 4 \%}$ for Stage I CRC, $\mathbf{9 4 . 0 6 \%}$ for Stage II CRC, $\mathbf{9 3 . 6 2 \%}$ for Stage III CRC, and $\mathbf{9 3 . 5 4 \%}$ for total colorectal tumors with a specificity of $\mathbf{9 4 . 0 5 \%}$. To our knowledge, this is the first study for colorectal neoplasia-specific methylation biomarkers based on CTCF-binding sites. Using a similar strategy, CTCF-binding sites could be potentially developed into biomarkers for other tumors. In summary, this study opens a new area in developing biomarkers for tumor prevention and treatment. 


\section{INTRODUCTION}

Colorectal cancer (CRC) is the third most commonly diagnosed cancer in males and second in females [1]. DNA methylation, which is one of the most important mechanisms involved in gene and microRNA expression regulation and in alternative gene splicing, plays important roles in the early stage of cancer [2]. Because it is stable and easily detected qualitatively or quantitatively, DNA methylation has been considered as the most promising diagnostic marker for early detection of cancer [3]. Over the recent years, many DNA methylation markers have been proposed as useful early detection markers for CRC [2, 4-14]. For example, the development of blood-based cancer detection tests improves patient compliance, thereby increasing the incidence of cancer detection at earlier stages. Currently, the most established methylated DNA blood biomarker is methylated septin 9 (SEPT9) $[15,16]$. SEPT9 is now commercially offered as a blood-based screening test in various assays. However, methylated SEPT9 has a limited sensitivity for the detection of advanced adenomas (11\%), underscoring the need for further improvement of this test for its implementation in population-based screening of colorectal neoplasia [17]. In addition, a stool DNA test (sDNA test), which includes quantitative molecular assays for $K R A S$ (kirsten rat sarcoma viral oncogene) mutations, aberrant NDRG4 (N-myc downstream-regulated 4) and $B M P 3$ (bone morphogenetic protein 3) methylation, and a hemoglobin immunoassay $[5,6,13]$, has culminated in the development of an FDA approved, clinically available stool-based CRC screening test [2]. However, its sensitivity for detecting advanced precancerous lesions was only $42.4 \%(P<0.001)$ [13]. In summary, although the impressive accuracy for CRC detection has been achieved, both serum-based DNA test and stool-based DNA test are currently less sensitive for CRC precursor lesions, particularly for adenomas [4-6, 13]. Therefore, urgent development of novel and robust detective biomarkers for the early detection of adenomas and CRC is required. Previously reported methylation biomarkers were primarily based on the methylation of gene promoters, but this study aimed to develop biomarkers with DNA binding sites of a multifunctional transcriptional factor CTCF (CCCTC-binding factor) for early detection of CRC. CTCF is a versatile transcription regulator that is ubiquitously expressed and evolutionarily conserved from the fruit fly to humans [18]. CTCF contains a highly conserved DNA-binding domain with 11 zinc fingers, and it binds to different DNA sequences by the combinatorial use of 11-zinc fingers to play a key role in many chromatin insulation events and epigenetic processes [19-26]. Despite its obvious importance, to our knowledge, there is no report considering CTCF-binding sites as biomarkers for the early detection of tumors. We considered CTCF- binding sites as potential biomarkers for early detection of CRC because of the following reasons: (i) CTCFbinding sites are widespread in human genome and CTCF occupancy at its binding sites is generally methylationsensitive [27-29]. (ii) Aberrant DNA methylation of CTCF-binding sites has also been connected with multiple malignancies [21, 30-35]. (iii) CTCF is involved in the maintenance of genomic methylation patterns [36, 37]. (iv) Immortalized cancer cell lines contain cell type-specific CTCF-binding pattern, which is significantly associated with differential methylation status [29]. Therefore, the methylation status of CTCF-binding sites is a potentially ideal biomarker for early detection of CRC for tumor prevention and treatment.

By genome-wide identification of candidate CTCFbinding sites using the combined analyses of genomewide occupation and methylation profile of CTCFbinding site with published genome-wide data, followed by stepwise screening and verification using methylationsensitive high-resolution melting (MS-HRM) and mass spectrometry analysis, this study aimed to (1) determine the performance of CTCF-binding sites in discriminating tumor tissues from normal tissues, (2) assess the potential of CTCF-binding sites as biomarkers for early-detection of CRC, and (3) provide a valuable reference to develop new biomarkers for the prevention and treatment of other tumors.

\section{RESULTS}

\section{MS-HRM analysis identified 23 CTCF-binding sites with high tumor specificity from the $\mathbf{1 2 1}$ candidate CTCF-binding sites}

By using different dilutions of fully methylated DNA against unmethylated DNA, with ratios of methylated DNA as $0,1,10$, and $100 \%$ methylation, we showed that methylation levels as low as $1 \%$ could be easily detected at the annealing temperature of $58^{\circ} \mathrm{C}$ by MS-HRM analysis (Figure 1). For the 121 candidate CTCF-binding sites, MS-HRM analysis identified 23 CTCF-binding sites with methylation profiles highly specific in tumor tissues and were, thus, considered as potential candidates for colorectal neoplasia biomarkers (Supplementary Table 1); the remaining 98 CTCF-binding sites were eliminated because they did not exhibit impressive tumor-specific methylation profiles. The methylation pattern of these 23 CTCF-binding sites was specific in $64 \%-94 \%$ of the tested tumor tissues. Among the 23 CTCF-binding sites, methylation levels of 16 sites were significantly higher in tumor tissues than those in matched normal counterparts $(\mathrm{T}>\mathrm{N})$. For the remaining seven sites, methylation levels were significantly lower in tumor tissues than those in matched normal counterparts $(\mathrm{T}<\mathrm{N})$. 


\section{Mass spectrometry analysis further screened out 5 final biomarkers with impressive detection accuracy from the 23 CTCF-binding sites}

To accurately quantify the DNA methylation differences between normal and tumor tissues, to evaluate the performance of different CTCF-binding sites in discriminating tumor tissues from normal tissues, the methylation state of the 23 tumor-specific CTCF- binding sites identified by MS-HRM analysis was further determined by mass spectrometry analysis in 20 pairs of normal and tumor tissues. Twenty tumor tissues comprised seven adenomas, six Stage I CRC, and seven Stage II CRC.

To determine the clustering profile in the overall methylation patterns of the samples and CTCF-binding sites, a hierarchical two-dimensional unsupervised clustering was performed between a set of 23 tumor-
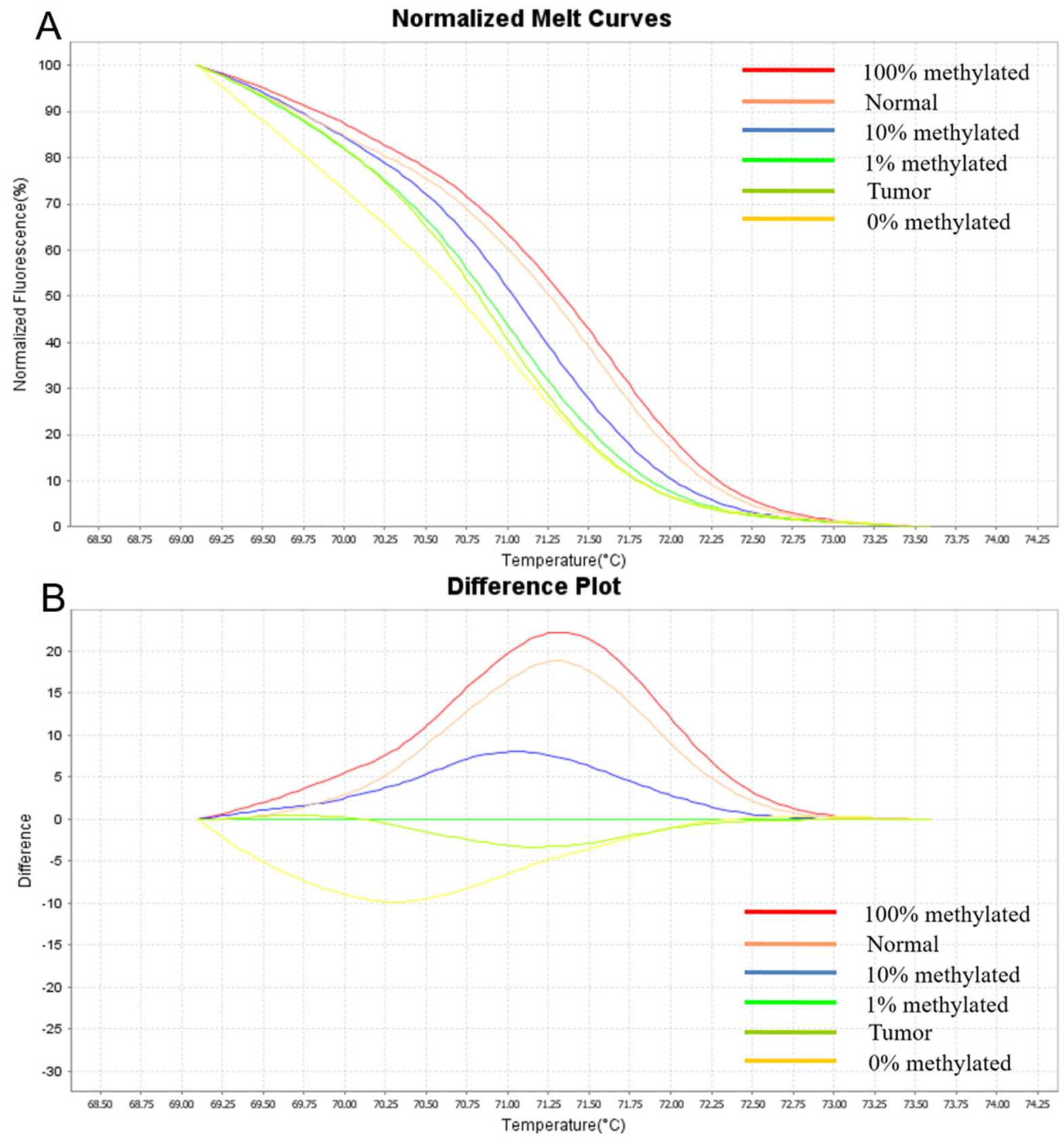

Figure 1: (A) Normalized melting profile. (B) Difference plots. The HRM standard melting curve was derived from five samples, with the following ratios of methylated DNA: $0,1,10$, and $100 \%$ methylation. At the annealing temperature of $58^{\circ} \mathrm{C}$, methylation levels as low as $1 \%$ could be easily detected. As shown in the figure, the methylation level of a representative CTCF-binding site in a tumor tissue is between $0-1 \%$, and its methylation level in the matched normal counterpart is between $10-100 \%$, indicating that the methylation level of this CTCF-binding site is significantly lower in tumor tissues than that in matched normal counterparts. 
specific CTCF-binding sites and a set of 40 colorectal tissue samples (Figure 2). All of the 40 colorectal samples were divided into two clusters (cluster $\mathrm{N}$ and cluster $\mathrm{T}$ ). Cluster $\mathrm{N}$ consisted of 20 normal tissues and two tumor tissues. Cluster $\mathrm{T}$ included the remaining 18 tumor tissues. In addition, mass spectrometry analysis confirmed methylation patterns of the 23 CTCF-binding sites identified by MS-HRM analysis, indicating that methylation levels of 16 sites were significantly higher in tumor tissues than those in the matched normal counterparts and the methylation levels of the remaining seven sites were significantly lower in tumor tissues than those in matched normal counterparts. The clustering results clearly showed that the 23 CTCF-binding sites could discriminate tumor tissues from normal colorectal tissues. We will next try to determine the ability of each CTCF-binding site to discriminate tumor tissues from normal tissues.
To find the most robust candidate biomarkers for the early detection of colorectal neoplasia, we used ROC curves to quantify the accuracy and robustness of each CTCF-binding site. In this analysis, the negative class consisted of 20 normal colorectal samples, and the positive class included 20 colorectal tumor samples. The discriminatory powers of 23 CTCF-binding sites are summarized in Table 1. Despite the relatively lower sample number in this analysis, the discriminatory powers of the $18 \mathrm{CTCF}$-binding sites were statistically significant $(P<0.0001)$. Nineteen sites exhibited a very robust discriminatory power with an AUC of $>0.8$. The sensitivity of these strong markers ranged between $50 \%$ and $97.37 \%$ at a specificity level of $95 \%$. According to the discriminatory power of individual CTCF-binding sites and the manual review of the discriminatory power for samples from early stage colorectal neoplasia (adenomas and Stage I CRC), ten CTCF-binding sites were selected

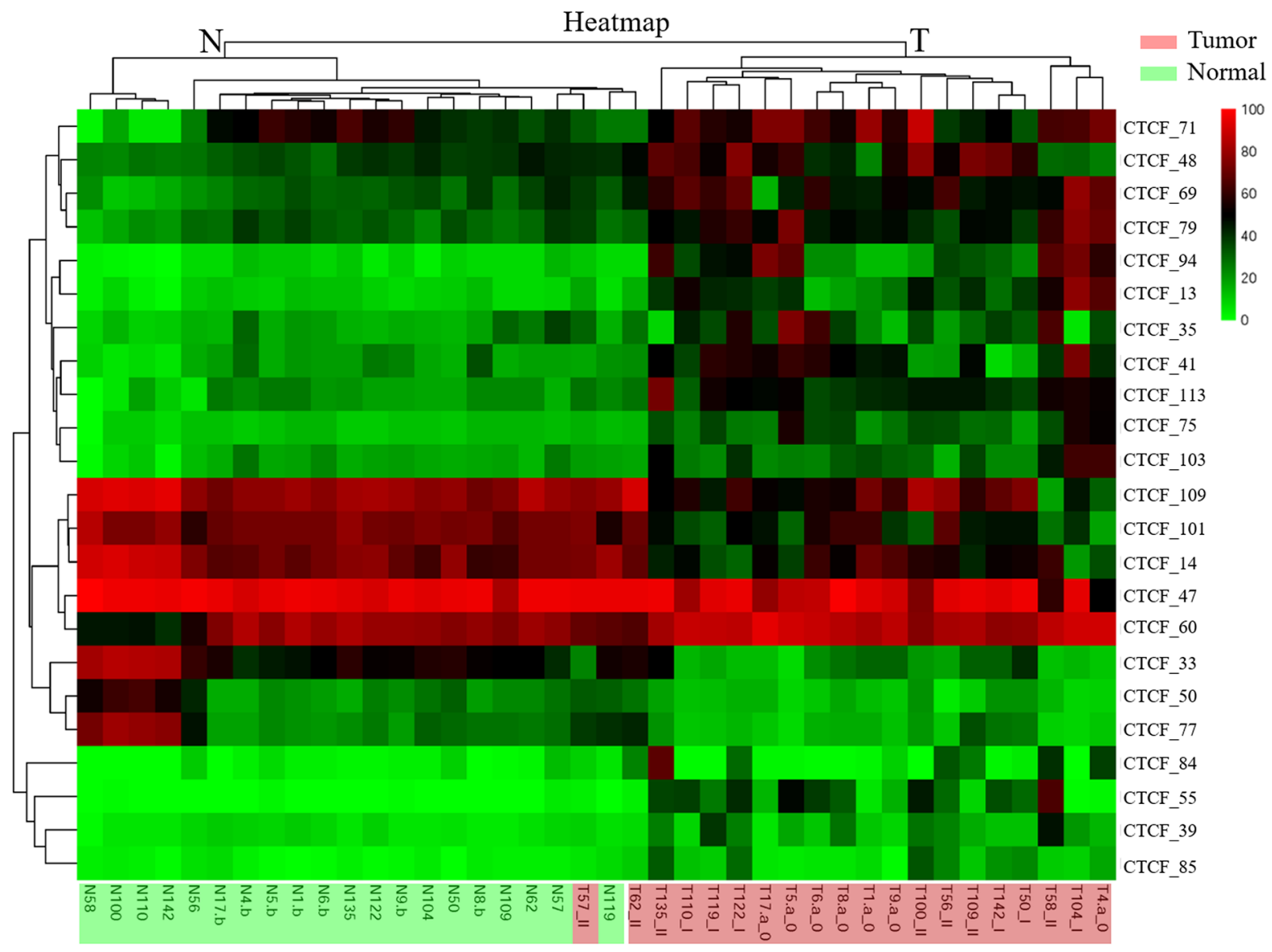

Figure 2: Hierarchical clustering of all 40 colorectal tissue samples and 23 tumor-specific CTCF-binding sites identified by MS-HRM analysis. Columns, patient samples; rows, tumor-specific CTCF-binding sites. Column annotations include the names of the CTCF-binding sites, row annotations include the names of the sample (N represents normal tissue; $\mathrm{T}$ represents tumor tissue) and tumor stages for tumor tissues ( 0 represents adenomas; I represents Stage I; II represents Stage II). The class information was unknown to the clustering algorithm. The average degree of methylation of each CTCF-binding site in each sample is represented by the decadic logarithm of the methylation proportion ranging from $0 \%$ methylated alleles (green) to methylation of all alleles (red). There are two main tissue clusters labeled as $\mathrm{N}$ and T. Cluster $\mathrm{N}$ consists of 18 normal tissues and two tumor tissues. Cluster $\mathrm{T}$ is composed of the remaining 18 tumor tissues. 
Table 1: Single-marker performance of the 23 candidate CTCF-binding sites

\begin{tabular}{|c|c|c|c|}
\hline CTCF site & $\mathbf{A U C}$ & $\boldsymbol{P}$ & Sensitivity at $95 \%$ specificity \\
\hline CTCF_13 & 0.997 & $<0.0001$ & $97.37[82.46,100.0]$ \\
\hline CTCF_113 & 0.995 & $<0.0001$ & $95.00[75.00,100.0]$ \\
\hline CTCF_75 & 0.993 & $<0.0001$ & $95.95[75.99,100.0]$ \\
\hline CTCF_55 & 0.984 & $<0.0001$ & $80.00[50.00,95.00]$ \\
\hline CTCF_79 & 0.961 & $<0.0001$ & $80.00[55.00,95.00]$ \\
\hline CTCF_94 & 0.956 & $<0.0001$ & $85.00[60.00,95.00]$ \\
\hline CTCF_33 & 0.954 & $<0.0001$ & $84.21[54.49,94.74]$ \\
\hline CTCF_69 & 0.946 & $<0.0001$ & $89.47[62.38,100.0]$ \\
\hline CTCF_14 & 0.92 & $<0.0001$ & $68.42[36.84,84.21]$ \\
\hline CTCF_101 & 0.917 & $<0.0001$ & $71.11[43.97,88.89]$ \\
\hline CTCF_85 & 0.916 & $<0.0001$ & $67.50[40.00,87.50]$ \\
\hline CTCF_39 & 0.907 & $<0.0001$ & $75.00[55.00,90.00]$ \\
\hline CTCF_103 & 0.891 & $<0.0001$ & $50.00[25.00,80.00]$ \\
\hline CTCF_109 & 0.879 & $<0.0001$ & $70.00[45.00,85.10]$ \\
\hline CTCF_50 & 0.866 & $<0.0001$ & $63.16[36.84,84.21]$ \\
\hline CTCF_41 & 0.838 & $<0.0001$ & $68.42[47.37,89.47]$ \\
\hline CTCF_77 & 0.828 & $<0.0001$ & $65.00[45.00,85.00]$ \\
\hline CTCF_60 & 0.826 & $<0.0001$ & $55.00[30.00,80.00]$ \\
\hline CTCF_48 & 0.809 & 0.0001 & $63.16[36.84,82.88]$ \\
\hline CTCF_35 & 0.781 & 0.0012 & $38.33[03.52,65.88]$ \\
\hline CTCF_71 & 0.754 & 0.0012 & $36.84[10.53,57.89]$ \\
\hline CTCF_47 & 0.724 & 0.0065 & $25.00[0.77,45.00]$ \\
\hline CTCF_84 & 0.63 & 0.1561 & $35.00[15.00,60.00]$ \\
\hline
\end{tabular}

NOTE: Shown is the AUC, P value, and sensitivity at 95\% specificity (median value plus $90 \%$ confidence interval estimated by bootstrapping) for each marker.

for further screening (marked in bold in Table 1). Of these ten CTCF-binding sites, the methylation levels of five sites in tumor tissues were significantly lower than those in normal tissues.

To reinforce the discriminatory power of our methylation biomarkers for adenomas, the methylation level of the 10 CTCF-binding sites was further determined in 90 samples by increasing the proportion of adenoma samples (20 normal tissues, 57 adenomas, six Stage I CRC, and seven Stage II CRC) by mass spectrometry analysis. The discriminatory powers of these 10 sites are summarized in Table 2. In this analysis, all of the 10 sites were statistically significant $(P<0.0001)$ and exhibited a very strong class separation with an AUC of $\geq 0.85$. The sensitivity of these strong markers ranged between $44.64 \%$ and $88.89 \%$ at a specificity level of $95 \%$. We strove to identify a panel as small as possible that would accurately identify colorectal tumors in the early stage to provide the most cost-effective screening method for early detection of colorectal tumors. Therefore, according to their discriminatory performance (AUC value), five CTCF-binding sites (marked in bold in Table 2) were selected as final biomarkers for further validation in a large sample set. Among these five CTCF-binding sites, the methylation level of one site (CTCF 33) in the tumor tissues was significantly lower than that in normal tissues. For the remaining four sites, methylation levels in tumor tissues were significantly higher than those in normal tissues. The discriminatory powers of these five sites were very strong with an AUC of $\geq 0.916$, and the sensitivity ranged between $73.08 \%$ and $88.89 \%$ at a specificity level of $95 \%$. 
Table 2: Single-marker performance of the 10 candidate CTCF-binding sites

\begin{tabular}{lccc}
\hline CTCF site & AUC & $\boldsymbol{P}$ & Sensitivity at 95\% specificity \\
\hline CTCF_113 & $\mathbf{0 . 9 7 7}$ & $<0.0001$ & $88.89[68.45,96.83]$ \\
CTCF_13 & $\mathbf{0 . 9 5 3}$ & $<0.0001$ & $80.08[54.75,96.95]$ \\
CTCF_33 & $\mathbf{0 . 9 4 3}$ & $<0.0001$ & $80.47[66.85,89.24]$ \\
CTCF_55 & $\mathbf{0 . 9 3 4}$ & $<0.0001$ & $80.77[65.58,90.68]$ \\
CTCF_94 & $\mathbf{0 . 9 1 6}$ & $<0.0001$ & $73.08[60.38,82.50]$ \\
CTCF_79 & 0.906 & $<0.0001$ & $74.46[61.17,83.69]$ \\
CTCF_101 & 0.881 & $<0.0001$ & $44.64[28.57,75.00]$ \\
CTCF_14 & 0.868 & $<0.0001$ & $59.38[37.81,75.62]$ \\
CTCF_77 & 0.863 & $<0.0001$ & $74.23[59.50,84.69]$ \\
CTCF_50 & 0.85 & $<0.0001$ & $54.71[38.26,74.98]$ \\
\hline
\end{tabular}

\section{Discriminatory performance of the five final biomarkers was reconfirmed with a large sample set by mass spectrometry analysis}

\section{Individual marker performance}

The discriminatory performance of the five $\mathrm{CTCF}$ binding sites selected as final biomarkers were further validated in a large sample set by mass spectrometry. The methylation level of these five CTCF-binding sites was examined in a large sample set (379 samples in total) with a negative class of 84 colorectal-derived normal tissues. The positive class was composed of 295 colorectal-derived tumor tissues from adenomas (108), Stage I CRC (39), Stage II CRC (101), and Stage III CRC (47). We analyzed the individual marker performance of the five final sites in adenomas, Stage I CRC, Stage II CRC, Stage III CRC, and total colorectal-derived tumor tissues, respectively (Table 3). As shown in Table 3, regardless of the adenoma, Stage I CRC, Stage II CRC, or Stage III CRC, all of the five markers were statistically significant $(P<0.0001)$ and showed a very strong individual performance with an AUC of $\geq 0.853$. The sensitivity of these five markers ranged between $57.95 \%$ and $96.84 \%$ at a specificity level of $95 \%$. In particular, for adenomas, all of the five markers showed a very strong individual performance with an AUC of $\geq 0.917$, and the sensitivity ranged between $73.52 \%$ and $82.62 \%$ at a specificity level of $95 \%$. All markers exhibited a very strong individual performance with an AUC of $\geq 0.891$ for all colorectal-derived tumor tissues, and the sensitivity ranged between $68.68 \%$ and $87.14 \%$ at a specificity level of $95 \%$.

We next compared the discriminatory power of these five new markers with two currently acknowledged robust methylation biomarkers for the early detection of colorectal tumors (BMP3 and NDRG4) in the same sample set. BMP3 and NDRG4 are methylation biomarkers of the
sDNA test, which has culminated in the development of an FDA-approved and clinically available stool-based CRC screening test $[2,13]$. One hundred and twenty colorectal samples (including 58 normal tissues, 46 adenomas, four Stage I CRC, eight Stage II CRC, and four Stage III CRC) were selected from the large sample set, and the methylation status of BMP3 and NDRG4 was determined by mass spectrometry. ROC curves were used to compare the accuracy and robustness of these seven markers (five new markers and BMP3 and NDRG4; Table 4). Although all the seven markers were statistically significant $(P<$ 0.0001 ), each of the five new markers exhibited a more robust discriminatory performance than that by $B M P 3$ or NDRG4. As shown in Table 4, the five new markers had an AUC of $\geq 0.89$, and the sensitivity ranged between $73.83 \%$ and $86.72 \%$ at a specificity level of $90 \%$, whereas $B M P 3$ had an AUC of 0.79 and the sensitivity was $48.56 \%$ at a specificity level of $90 \%$ and NDRG4 had an AUC of 0.88 with the sensitivity of $69.56 \%$ at a specificity level of $90 \%$. In particular, the sensitivity of four of the five new markers was over $81 \%$ at a specificity level of $90 \%$, but both $B M P 3$ and NDRG4 exhibited less than $70 \%$ sensitivity, which is a further indication that the discriminatory accuracy of our new markers is significantly higher than that of $B M P 3$ or NDRG4.

\section{Marker panel performance}

When we considered the five final CTCF-binding sites as a panel of markers for the early-detection of colorectal tumors, the discriminatory performance improved significantly compared to that of the best single marker. Marker panel performance for different colorectal tumor stages is summarized in Table 5. When tumorpositive was defined as having two or more (of the five) positive CTCF-binding sites, the marker panel yielded the best sensitivity and specificity (Table 5). In this situation, the sensitivity of the marker panel was $91.67 \%$ 
Table 3: The discriminatory performance of the five final CTCF-binding sites in the large sample set

\begin{tabular}{|c|c|c|c|c|c|c|c|c|c|c|c|c|c|c|c|}
\hline \multirow[b]{2}{*}{ CTCF site } & \multicolumn{3}{|c|}{ Adenoma $(\mathrm{N}=108)$} & \multicolumn{3}{|c|}{ Stage I (N=39) } & \multicolumn{3}{|c|}{ Stage II (N=101) } & \multicolumn{3}{|c|}{ Stage III $(N=47)$} & \multicolumn{3}{|c|}{ Total $(\mathrm{N}=295)$} \\
\hline & AUC & $P$ & $\begin{array}{c}\text { Sensitivity } \\
\text { at } 95 \% \\
\text { specificity }\end{array}$ & AUC & $P$ & $\begin{array}{c}\text { Sensitivity } \\
\text { at } 95 \% \\
\text { specificity }\end{array}$ & AUC & $P$ & $\begin{array}{c}\text { Sensitivity } \\
\text { at } 95 \% \\
\text { specificity }\end{array}$ & AUC & $P$ & $\begin{array}{c}\text { Sensitivity } \\
\text { at } 95 \% \\
\text { specificity }\end{array}$ & AUC & $P$ & $\begin{array}{c}\text { Sensitivity } \\
\text { at } 95 \% \\
\text { specificity }\end{array}$ \\
\hline CTCF_33 & 0.93 & $<0.0001$ & $\begin{array}{c}80.51 \\
{[68.94,88.37]}\end{array}$ & 0.9 & $<0.0001$ & $\begin{array}{c}67.47 \\
{[49.41,83.45]}\end{array}$ & 0.9 & $<0.0001$ & $\begin{array}{c}57.59 \\
{[46.78,69.11]}\end{array}$ & 0.9 & $<0.0001$ & $\begin{array}{c}65.22 \\
{[47.83,80.43]}\end{array}$ & 0.9 & $<0.0001$ & $\begin{array}{c}68.68 \\
{[60.17,76.25]}\end{array}$ \\
\hline CTCF_55 & 0.93 & $<0.0001$ & $\begin{array}{c}73.52 \\
{[64.75,82.33]}\end{array}$ & 1 & $<0.0001$ & $\begin{array}{c}95.13 \\
{[82.07,100.0]}\end{array}$ & 1 & $<0.0001$ & $\begin{array}{c}88.87 \\
{[59.14,95.88]}\end{array}$ & 0.9 & $<0.0001$ & $\begin{array}{c}80.77 \\
{[63.92,91.52]}\end{array}$ & 0.9 & $<0.0001$ & $\begin{array}{c}84.66 \\
{[76.19,88.87]}\end{array}$ \\
\hline CTCF_94 & 0.92 & $<0.0001$ & $\begin{array}{c}79.44 \\
{[70.09,86.61]}\end{array}$ & 1 & $<0.0001$ & $\begin{array}{c}83.59 \\
{[61.83,92.31]}\end{array}$ & 0.9 & $<0.0001$ & $\begin{array}{c}74.46 \\
{[63.37,82.24]}\end{array}$ & 0.9 & $<0.0001$ & $\begin{array}{c}74.04 \\
{[47.92,87.41]}\end{array}$ & 0.9 & $<0.0001$ & $\begin{array}{c}77.41 \\
{[70.09,84.04]}\end{array}$ \\
\hline CTCF_113 & 0.92 & $<0.0001$ & $\begin{array}{c}82.62 \\
{[72.41,89.91]}\end{array}$ & 1 & $<0.0001$ & $\begin{array}{c}92.92 \\
{[81.76,98.57]}\end{array}$ & 1 & $<0.0001$ & $\begin{array}{c}89.19 \\
{[81.98,94.63]}\end{array}$ & 0.9 & $<0.0001$ & $\begin{array}{c}89.36 \\
{[76.60,97.87]}\end{array}$ & 0.9 & $<0.0001$ & $\begin{array}{c}85.17 \\
{[79.23,90.01]}\end{array}$ \\
\hline CTCF_13 & 0.93 & $<0.0001$ & $\begin{array}{c}79.79 \\
{[70.56,87.08]}\end{array}$ & 1 & $<0.0001$ & $\begin{array}{c}96.84 \\
{[77.68,100.0]}\end{array}$ & 1 & $<0.0001$ & $\begin{array}{c}88.06 \\
{[80.43,93.10]}\end{array}$ & 1 & $<0.0001$ & $\begin{array}{c}87.66 \\
{[73.66,95.94]}\end{array}$ & 1 & $<0.0001$ & $\begin{array}{c}87.14 \\
{[81.77,91.31]}\end{array}$ \\
\hline
\end{tabular}

for adenomas, $97.44 \%$ for Stage I CRC, $94.06 \%$ for Stage II CRC, 93.62\% for Stage III CRC, and 93.54\% for total colorectal tumors at a specificity level of $94.05 \%$.

\section{DISCUSSION}

By genome-wide identification of candidate CTCF-binding sites using the combination of genome-wide occupation and methylation profile analyses of CTCF-binding site, followed by stepwise screening and verification using MS-HRM and mass spectrometry analysis, this study identified a set of colorectal neoplasia-specific methylation biomarkers with robust performance in diverse sample sets. The top five biomarkers, named as CTCF_13, CTCF_33, CTCF_55, CTCF_94, and CTCF_113, were selected and recommended as biomarkers for early detection of colorectal neoplasia. Compared to the performance of two currently acknowledged robust methylation biomarkers (BMP3 and NDRG4) in the same sample set, all of the five new markers exhibited a more robust discriminatory performance. In addition, when the five new biomarkers were considered as a marker panel and tumor-positive was defined as having two or more (of the five) positive biomarkers, the discriminatory performance improved significantly compared to that by the best single marker.

Although many biomarkers based on DNA methylation of gene promoters have been proposed as useful early detection markers for CRC [2, 4-14], the accuracy of these biomarkers still needs to be improved, particularly with regard to the detection accuracy of adenomas. Currently, the most established methylated DNA blood biomarker is methylated SEPT9. SEPT9 is now commercially offered as a blood-based screening test in various assays. Furthermore, the sensitivity and specificity of the SEPT9 assay for CRC was comparable with that of the conventional FOBT, confirming its potential usefulness as a blood-based biomarker for CRC [16]. However, a recent study demonstrated that the methylated SEPT9 assay was superior to FIT at detecting
CRC neoplasms, but both approaches were suboptimal for diagnosing patients with advanced adenomas [17], which underscored the need for further improvement of this test for implementation of a population-based screening of colorectal neoplasia. In addition, although the sDNA test has culminated in the development of an FDA approved, clinically available stool-based CRC screening test, its sensitivity for detecting advanced precancerous lesions is only $42.4 \%$ [13]. In summary, although impressive accuracy for CRC detection has been achieved based on DNA methylation biomarkers, both serum-based DNA test and stool-based DNA test were less sensitive for CRC precursor lesions, particularly for adenomas [4-6, 13]. However, this study identified a set of colorectal neoplasia-specific methylation biomarkers, viz., CTCF_33, CTCF_55, CTCF_94, CTCF_113, and $\mathrm{CTCF}_{-} 13$, with high accuracy both for colorectal adenoma and CRC. Notably, as the only marker whose methylation level in tumor tissues was significantly lower than that in normal tissues, CTCF_33 was more sensitive for adenoma than for CRC (Table 3 ). In particular, when the five new biomarkers were considered as a marker panel and tumor-positive was defined as having two or more (of the five) positive biomarkers, the marker panel could achieve a sensitivity of $91.67 \%$ for adenomas with a specificity of $94.05 \%$. In other words, compared with the methylated SEPT9 assay and the sDNA test, our new marker panel exhibited significantly higher accuracy for adenoma and afforded sensitivity and specificity of $>90 \%$ both for adenoma and CRC (Table 5). In addition, although the sDNA test may be more accurate than the methylated SEPT9 assay in detecting colorectal neoplasia [5], it includes quantitative molecular assays for $K R A S$ mutations, aberrant NDRG4 and BMP3 methylation, and a hemoglobin immunoassay. By comparison, our new test only required detecting the aberrant methylation of five CTCF-binding sites, indicating that it is more labor- and cost-effective, which is very important for the implementation of this test for population-based screening of colorectal neoplasia. 
Table 4: Comparison of new markers with existing markers

\begin{tabular}{lccc}
\hline Marker & AUC & $\boldsymbol{P}$ & Sensitivity at 90\% specificity \\
\hline CTCF_33 & 0.9 & $<0.0001$ & $81.69[61.51,93.23]$ \\
CTCF_55 & 0.92 & $<0.0001$ & $81.02[68.86,88.35]$ \\
CTCF_94 & 0.89 & $<0.0001$ & $73.83[56.97,85.06]$ \\
CTCF_113 & 0.92 & $<0.0001$ & $86.72[73.32,95.00]$ \\
CTCF_13 & 0.93 & $<0.0001$ & $81.64[67.75,92.07]$ \\
BMP3 & 0.79 & $<0.0001$ & $48.56[32.06,61.96]$ \\
NDRG4 & 0.88 & $<0.0001$ & $69.56[50.94,82.21]$ \\
\hline
\end{tabular}

NOTE: Shown is the AUC, P value, and sensitivity at $90 \%$ specificity (median value plus $90 \%$ confidence interval estimated by bootstrapping) for each marker.

Table 5: Marker panel performance

\begin{tabular}{lcccccc}
\hline \multirow{2}{*}{ No. of CTCF site } & \multicolumn{5}{c}{ Sensitivity (\%) } & \multirow{2}{*}{ Specificity (\%) } \\
\cline { 2 - 5 } & Adenoma & Stage I & Stage II & Stage III & Total & 79.76 \\
$\geq 1$ & 96.30 & 97.44 & 98.02 & 95.74 & 97.28 & $\mathbf{9 4 . 0 5}$ \\
$\geq \mathbf{2}$ & $\mathbf{9 1 . 6 7}$ & $\mathbf{9 7 . 4 4}$ & $\mathbf{9 4 . 0 6}$ & $\mathbf{9 3 . 6 2}$ & $\mathbf{9 3 . 5 4}$ & 97.62 \\
$\geq 3$ & 85.19 & 92.31 & 91.09 & 87.23 & 89.8 & 100 \\
$\geq 4$ & 74.07 & 84.62 & 75.25 & 65.96 & 74.49 & 100 \\
5 & 50.93 & 56.41 & 40.59 & 51.06 & 47.96 & \\
\hline
\end{tabular}

Furthermore, compared with the existing biomarkers that are based on DNA methylation in the promoter region, biomarkers based on the methylation state of CTCFbinding sites showed many other advantages: (1) CTCFbinding sites are widespread in human genome and most of their locations on human chromosome are already known, facilitating genome-wide screening and validation of biomarkers for tumor prevention and treatment. (2) Compared with $\mathrm{CpG}$ islands in the promoter region, which are approximately $200 \mathrm{bp}$ in length, CTCF-binding sites only have a sequence length of around $30 \mathrm{bp}$ [46], which is more conducive to the development of DNA methylation biomarkers with high detection sensitivity and accuracy. This is particularly true for serum- and stoolbased DNA tests for the early detection of CRC because tumor DNA content in blood or stool samples is very low and is degraded, and tumor DNA is further degraded after bisulfite treatment. In this situation, methylation biomarkers with shorter target sequences will be more advantageous. (3) Aberrant DNA methylation of CTCFbinding sites has been connected for all types of tumors; therefore, using a strategy similar to that described in our study, CTCF-binding sites could be potentially developed into biomarkers for the prevention and treatment of other tumors.
To the best of our knowledge, this is the first study for colorectal neoplasia-specific methylation biomarkers based on CTCF DNA-binding sites in a screening setting. Moreover, these biomarkers may be useful in tumor prevention and treatment with respect to factors such as early detection, diagnosis, tendency of tissue invasion, metastasis, prognosis, and response to chemotherapy. Using a strategy similar to that of this study, CTCF DNA-binding sites could be potentially developed into biomarkers for other tumors. In summary, this study presents a novel strategy to develop biomarkers for tumor prevention and treatment based on CTCF DNA-binding sites. We hope that our study will serve as a starting point for more extensive and in-depth studies to fully estimate the potential of CTCF DNA-binding sites as methylation biomarkers for clinical applications in tumor prevention and treatment.

\section{MATERIALS AND METHODS}

\section{Tissue samples}

Two hundred and ninety-five fresh colorectal tumor tissues and eighty-four matched normal tissues were obtained from surgical resections, fresh-frozen, and 
stored at $-80^{\circ} \mathrm{C}$. Samples of normal tissue were obtained from a distance of at least $6 \mathrm{~cm}$ from the tumor and were confirmed to be tumor-free by microscopy. These patients had undergone curative surgery at the First Affiliated Hospital of Kunming Medical University between 2014 and 2016. Patients gave informed consent in writing to use their bowel tissue for research. The selection was solely based on the availability of tissues for the study, and we did not exclude patients with a family history of CRCs. Clinicopathological information, including age, sex, tumor location, and tumor stage, were obtained from these patients (Supplementary Table 2). Tumors were staged on the basis of the pathological tumor-node-metastasis (pTNM) staging system of the American Joint Committee on Cancer (AJCC). The study was approved by the First Affiliated Hospital of Kunming Medical University Ethics Committee.

\section{Candidate CTCF-binding site discovery}

Wang et al. analyzed genome-wide occupancy patterns of CTCF in 19 diverse human cell types, including seven immortal cell lines and 12 normal cell types [29]. They identified 4146 specific CTCF-binding sites whose occupancy was distinguished in immortal cell lines, epithelia, fibroblasts, and endothelia. Among the 4146 CTCF-binding sites, the occupancy of 1236 sites are specific only in immortal cell lines, and these binding sites can distinguish immortal cell lines from normal cell lines. By analyzing the association data between genomewide occupancy and the methylation state of these 1236 CTCF-binding sites in 13 cell lines (including six immortal cell lines and seven normal cell lines) published by Wang et al. [29], we identified 121 CTCF-binding sites whose occupancy was significantly associated with methylation. At these 121 sites, increased methylation was negatively associated with occupancy, indicating that the CTCF occupancy at these 121 sites is specific in cancer cells and is significantly associated with methylation. In theory, the DNA methylation status of these sites can be used to distinguish cancer cells from normal cells. We hypothesize that these $121 \mathrm{CTCF}$-binding sites can be used as candidate biomarkers to distinguish tumor tissues from normal tissues.

\section{DNA extraction and bisulfite modification}

Whole genome DNA was extracted using the QIAamp DNA Mini Kit (QIAGEN, Germantown, MD). Bisulfite modification was performed using an EpiTect Fast DNA Bisulfite Kit (QIAGEN, Germantown, MD) according to the manufacturer's instructions.

\section{MS-HRM analysis}

MS-HRM analysis is based on the comparison of the melting profiles of PCR products from unknown samples with profiles specific for PCR products derived from methylated and unmethylated control DNAs. The protocol consists of PCR amplification of bisulfitemodified DNA with primers designed to proportionally amplify both methylated and unmethylated templates and subsequent HRM analysis of the PCR product. Because it is a labor- and cost-efficient, high-throughput, and sensitive technology for single-locus methylation detection [38], we performed first screening of these 121 candidate CTCF-binding sites against 33 pairs of normal and tumor tissues using the MS-HRM approach to eliminate markers that did not show evidence of tumor-associated methylation. The thirty-three tumor tissues tested included six adenomas, nine Stage I CRC, 10 Stage II CRC, and eight Stage III CRC tissues. MSHRM PCR primers were designed for 121 candidate CTCF-binding sites according to their genomic DNA sequence [29] and their most possible binding positions [27]. The most possible CTCF-binding positions for each CTCF-binding site was predicted by using the online database CTCFBSDB 2.0 (http://insulatordb.uthsc.edu/), which now contains almost 15 million CTCF-binding sequences in 10 species [27]. MS-HRM PCR product for each CTCF-binding site must include the most possible CTCF-binding positions. We followed the MS-HRM primer design guidelines as previously outlined [38]. MS-HRM primers and chromosomal locations for 121 candidate CTCF-binding sites are listed in Supplementary Table 3. PCR amplification and HRM analysis were performed on an ABI StepOne Plus real-time PCR system (Applied Biosystems, USA) using MeltDoctor ${ }^{\mathrm{TM}}$ HRM Master Mix (Applied Biosystems, USA) according to the manufacturer's instructions.

A standard curve with known methylation ratios was included in each assay and was used to deduce the methylation ratio of each tumor and normal tissue. The HRM data were analyzed using the ABI high-resolution melting software (Applied Biosystems, USA). Output plots are in the form of normalized melting curves and difference plots.

\section{Quantitative high-throughput DNA methylation analysis by mass spectrometry}

Because MS-HRM only provides a semiquantitative measure of cytosines that have been converted to thymines and is only used for qualitative comparisons and not for quantitative analysis [38, 39], mass spectrometry was used to further screen and validate the CTCF-binding sites identified by MS-HRM with the SEQUENOM MassARRAY platform (Agena Bioscience Inc., San Diego, CA previously Sequenom Inc.) using the EpiTYPER ${ }^{\circledR} T$ Complete Reagent Kit as previously described [40, 41]. By comparing the performance of all widely used DNA methylation analysis methods that are compatible with routine clinical use, MassARRAY analysis has been 
confirmed to be a reliable method when validating DNA methylation differences in large cohorts and is also an excellent technology for developing epigenetic biomarkers [39]. MassARRAY is an accurate, sensitive, and reproducible high-throughput quantification technology for DNA methylation profile that is compatible with automation [40, 42]. Bisulfite-treated DNA was subjected to PCR and then processed following the manufacturer's instructions. The methylation status of a detected pattern was then analyzed using Epityper software version 1.2 (Agena Bioscience Inc., San Diego, CA, USA). PCR primers were designed by using the online tool EpiDesigner (www.EpiDesigner.com) (Agena Bioscience Inc., San Diego, CA, USA) and are listed in Supplementary Table 4. After the T-cleavage reaction, the resulting fragments of each CTCF-binding site may contain more than one $\mathrm{CpG}$ dinucleotide, and are, therefore, referred to as "CpG units." The CpG units that produced data for less than $30 \%$ of samples (unreliable $\mathrm{CpG}$ units) and samples lacking more than $30 \%$ of their data points (unreliable samples) were discarded [43]. The methylation status of each CTCFbinding site was represented by the $\mathrm{CpG}$ unit with the highest area under the curve (AUC) value according to a receiver operating characteristic (ROC) analysis.

\section{Statistical methods}

Hierarchical clustering analysis of the MassARRAY data was performed using the average method via $\mathrm{R}$ package pheatmap [44]. The distance matrix used for clustering was calculated using the R package vegan [45].

AUC values were estimated using MedCalc software (version 9.2.0.0; Broekstraat, Mariakerke, Belgium). Sensitivity and specificity were estimated by 1000 bootstrapping runs. We report sensitivity at the $90 \%$ or $95 \%$ specificity level from the 1000 bootstrap runs at $95 \%$ confidence intervals. $P<0.05$ was considered statistically significant. For the five-marker panel analysis, we considered two or more markers positive as tumor positive.

\section{ACKNOWLEDGMENTS}

We thank professor Guoqing Pan for his assistance in sample collections. We thank Professor Jun Yu and Qiuwei Pan for their comments and suggestions on an earlier draft of the manuscript.

\section{CONFLICTS OF INTEREST}

All authors disclose no potential conflicts of interest.

\section{FUNDING}

This work was supported by grants from National Natural Science Foundation of China (81660094), Yunnan
Applied Basic research Projects-Union Foundation of Kunming Medical University (2017FE467(-132)), China Postdoctoral Science Foundation (2015M572504), the Key Science and Technology Planning Project of Kunming Science and Technology Bureau, the China Guanghua Foundation and Medical Leading Talent of Yunnan Province (No. L-201205).

\section{REFERENCES}

1. Torre LA, Bray F, Siegel RL, Ferlay J, Lortet-Tieulent J, Jemal A. Global cancer statistics, 2012. CA Cancer J Clin. 2015; 65:87-108.

2. Okugawa Y, Grady WM, Goel A. Epigenetic alterations in colorectal cancer: emerging biomarkers. Gastroenterology. 2015; 149:1204-1225.e1212.

3. Laird PW. The power and the promise of DNA methylation markers. Nat Rev Cancer. 2003; 3:253-266.

4. Warren JD, Xiong W, Bunker AM, Vaughn CP, Furtado LV, Roberts WL, Fang JC, Samowitz WS, Heichman KA. Septin 9 methylated DNA is a sensitive and specific blood test for colorectal cancer. BMC Med. 2011; 9:133.

5. Ahlquist DA, Taylor WR, Mahoney DW, Zou H, Domanico M, Thibodeau SN, Boardman LA, Berger BM, Lidgard GP. The stool DNA test is more accurate than the plasma septin 9 test in detecting colorectal neoplasia. Clin Gastroenterol Hepatol. 2012; 10:272-277.

6. Ahlquist DA, Zou H, Domanico M, Mahoney DW, Yab TC, Taylor WR, Butz ML, Thibodeau SN, Rabeneck L, Paszat LF, Kinzler KW, Vogelstein B, Bjerregaard NC, et al. Next-generation stool DNA test accurately detects colorectal cancer and large adenomas. Gastroenterology. 2012; 142:248-256.

7. Draht MX, Riedl RR, Niessen H, Carvalho B, Meijer GA, Herman JG, van Engeland M, Melotte V, Smits KM. Promoter $\mathrm{CpG}$ island methylation markers in colorectal cancer: the road ahead. Epigenomics. 2012; 4:179-194.

8. Zou H, Allawi H, Cao X, Domanico M, Harrington J, Taylor WR, Yab T, Ahlquist DA, Lidgard G. Quantification of methylated markers with a multiplex methylation-specific technology. Clin Chem. 2012; 58:375-383.

9. Carmona FJ, Azuara D, Berenguer-Llergo A, Fernandez AF, Biondo S, de Oca J, Rodriguez-Moranta F, Salazar R, Villanueva A, Fraga MF, Guardiola J, Capella G, Esteller $\mathrm{M}$, et al. DNA methylation biomarkers for noninvasive diagnosis of colorectal cancer. Cancer Prev Res (Phila). 2013; 6:656-665.

10. Ladabaum U, Allen J, Wandell M, Ramsey S. Colorectal cancer screening with blood-based biomarkers: costeffectiveness of methylated septin 9 DNA versus current strategies. Cancer Epidemiol Biomarkers Prev. 2013; 22:1567-1576.

11. Lidgard GP, Domanico MJ, Bruinsma JJ, Light J, Gagrat ZD, Oldham-Haltom RL, Fourrier KD, Allawi H, Yab TC, 
Taylor WR, Simonson JA, Devens M, Heigh RI, et al. Clinical performance of an automated stool DNA assay for detection of colorectal neoplasia. Clin Gastroenterol Hepatol. 2013; 11:1313-1318.

12. Oh T, Kim N, Moon Y, Kim MS, Hoehn BD, Park CH, Kim TS, Kim NK, Chung HC, An S. Genome-wide identification and validation of a novel methylation biomarker, SDC2, for blood-based detection of colorectal cancer. J Mol Diagn. 2013; 15:498-507.

13. Imperiale TF, Ransohoff DF, Itzkowitz SH, Levin TR, Lavin P, Lidgard GP, Ahlquist DA, Berger BM. Multitarget stool DNA testing for colorectal-cancer screening. N Engl J Med. 2014; 370:1287-1297.

14. Shah R, Jones E, Vidart V, Kuppen PJ, Conti JA, Francis NK. Biomarkers for early detection of colorectal cancer and polyps: systematic review. Cancer Epidemiol Biomarkers Prev. 2014; 23:1712-1728.

15. Lofton-Day C, Model F, Devos T, Tetzner R, Distler J, Schuster M, Song X, Lesche R, Liebenberg V, Ebert M, Molnar B, Grutzmann R, Pilarsky C, et al. DNA methylation biomarkers for blood-based colorectal cancer screening. Clin Chem. 2008; 54:414-423.

16. Church TR, Wandell M, Lofton-Day C, Mongin SJ, Burger M, Payne SR, Castanos-Velez E, Blumenstein BA, Rosch T, Osborn N, Snover D, Day RW, Ransohoff DF, et al. Prospective evaluation of methylated SEPT9 in plasma for detection of asymptomatic colorectal cancer. Gut. 2014; 63:317-325.

17. Jin $P$, Kang Q, Wang X, Yang L, Yu Y, Li N, He YQ, Han X, Hang J, Zhang J, Song LL, Han Y, Sheng Q. Performance of a second-generation methylated SEPT9 test in detecting colorectal neoplasm. J Gastroenterol Hepatol. 2015; 30:830-833.

18. Moon H, Filippova G, Loukinov D, Pugacheva E, Chen Q, Smith ST, Munhall A, Grewe B, Bartkuhn M, Arnold R, Burke LJ, Renkawitz-Pohl R, Ohlsson R, et al. CTCF is conserved from Drosophila to humans and confers enhancer blocking of the Fab-8 insulator. EMBO Rep. 2005; 6:165-170.

19. West AG, Gaszner M, Felsenfeld G. Insulators: many functions, many mechanisms. Genes Dev. 2002; 16:271-288.

20. Bell AC, Felsenfeld G. Methylation of a CTCF-dependent boundary controls imprinted expression of the Igf2 gene. Nature. 2000; 405:482-485.

21. Hark AT, Schoenherr CJ, Katz DJ, Ingram RS, Levorse JM, Tilghman SM. CTCF mediates methylation-sensitive enhancer-blocking activity at the H19/Igf2 locus. Nature. 2000; 405:486-489.

22. Chao W, Huynh KD, Spencer RJ, Davidow LS, Lee JT. CTCF, a candidate trans-acting factor for X-inactivation choice. Science. 2002; 295:345-347.
23. Valley CM, Willard HF. Genomic and epigenomic approaches to the study of X chromosome inactivation. Curr Opin Genet Dev. 2006; 16:240-245.

24. Ling JQ, Li T, Hu JF, Vu TH, Chen HL, Qiu XW, Cherry AM, Hoffman AR. CTCF mediates interchromosomal colocalization between Igf2/H19 and Wsb1/Nf1. Science. 2006; 312:269-272.

25. Lieberman-Aiden E, van Berkum NL, Williams L, Imakaev M, Ragoczy T, Telling A, Amit I, Lajoie BR, Sabo PJ, Dorschner MO, Sandstrom R, Bernstein B, Bender MA, et al. Comprehensive mapping of long-range interactions reveals folding principles of the human genome. Science. 2009; 326:289-293.

26. Dixon JR, Selvaraj S, Yue F, Kim A, Li Y, Shen Y, Hu M, Liu JS, Ren B. Topological domains in mammalian genomes identified by analysis of chromatin interactions. Nature. 2012; 485:376-380.

27. Ziebarth JD, Bhattacharya A, Cui Y. CTCFBSDB 2.0: a database for CTCF-binding sites and genome organization. Nucleic Acids Res. 2013; 41:D188-194.

28. Chen X, Xu H, Yuan P, Fang F, Huss M, Vega VB, Wong E, Orlov YL, Zhang W, Jiang J, Loh YH, Yeo HC, Yeo ZX, et al. Integration of external signaling pathways with the core transcriptional network in embryonic stem cells. Cell. 2008; 133:1106-1117.

29. Wang H, Maurano MT, Qu H, Varley KE, Gertz J, Pauli F, Lee K, Canfield T, Weaver M, Sandstrom R, Thurman RE, Kaul R, Myers RM, et al. Widespread plasticity in CTCF occupancy linked to DNA methylation. Genome Res. 2012; 22:1680-1688.

30. Yoon BJ, Herman H, Sikora A, Smith LT, Plass C, Soloway PD. Regulation of DNA methylation of Rasgrf1. Nat Genet. 2002; 30:92-96.

31. Butcher DT, Mancini-DiNardo DN, Archer TK, Rodenhiser DI. DNA binding sites for putative methylation boundaries in the unmethylated region of the BRCA1 promoter. Int $\mathrm{J}$ Cancer. 2004; 111:669-678.

32. Soto-Reyes E, Recillas-Targa F. Epigenetic regulation of the human $\mathrm{p} 53$ gene promoter by the CTCF transcription factor in transformed cell lines. Oncogene. 2010; 29:2217-2227.

33. Saldana-Meyer R, Gonzalez-Buendia E, Guerrero G, Narendra V, Bonasio R, Recillas-Targa F, Reinberg D. CTCF regulates the human p53 gene through direct interaction with its natural antisense transcript, Wrap53. Genes Dev. 2014; 28:723-734.

34. Mendez-Catala CF, Gretton S, Vostrov A, Pugacheva E, Farrar D, Ito Y, Docquier F, Kita GX, Murrell A, Lobanenkov V, Klenova E. A novel mechanism for CTCF in the epigenetic regulation of Bax in breast cancer cells. Neoplasia. 2013; 15:898-912.

35. Kemp CJ, Moore JM, Moser R, Bernard B, Teater M, Smith LE, Rabaia NA, Gurley KE, Guinney J, Busch SE, Shaknovich R, Lobanenkov VV, Liggitt D, et al. CTCF 
haploinsufficiency destabilizes DNA methylation and predisposes to cancer. Cell Rep. 2014; 7:1020-1029.

36. Guastafierro T, Cecchinelli B, Zampieri M, Reale A, Riggio G, Sthandier O, Zupi G, Calabrese L, Caiafa P. CCCTCbinding factor activates PARP-1 affecting DNA methylation machinery. J Biol Chem. 2008; 283:21873-21880.

37. Zampieri M, Guastafierro T, Calabrese R, Ciccarone F, Bacalini MG, Reale A, Perilli M, Passananti C, Caiafa P. ADP-ribose polymers localized on Ctcf-Parp1-Dnmt1 complex prevent methylation of Ctcf target sites. Biochem J. 2012; 441:645-652.

38. Wojdacz TK, Dobrovic A, Hansen LL. Methylationsensitive high-resolution melting. Nat Protoc. 2008; 3:1903-1908.

39. BLUEPRINT Consortium. Quantitative comparison of DNA methylation assays for biomarker development and clinical applications. Nat Biotechnol. 2016; 34:726-737.

40. Ehrich M, Nelson MR, Stanssens P, Zabeau M, Liloglou T, Xinarianos G, Cantor CR, Field JK, van den Boom D. Quantitative high-throughput analysis of DNA methylation patterns by base-specific cleavage and mass spectrometry. Proc Natl Acad Sci U S A. 2005; 102:15785-15790.

41. Koh YW, Chun SM, Park YS, Song JS, Lee GK, Khang SK, Jang SJ. Association between the $\mathrm{CpG}$ island methylator phenotype and its prognostic significance in primary pulmonary adenocarcinoma. Tumour Biol. 2016; 37:10675-10684.

42. Radpour R, Haghighi MM, Fan AX, Torbati PM, Hahn S, Holzgreve W, Zhong XY. High-throughput hacking of the methylation patterns in breast cancer by in vitro transcription and thymidine-specific cleavage mass array on MALDI-TOF silico-chip. Mol Cancer Res. 2008; 6:1702-1709.

43. Ollikainen M, Smith KR, Joo EJ, Ng HK, Andronikos R, Novakovic B, Abdul Aziz NK, Carlin JB, Morley R, Saffery $\mathrm{R}$, Craig JM. DNA methylation analysis of multiple tissues from newborn twins reveals both genetic and intrauterine components to variation in the human neonatal epigenome. Hum Mol Genet. 2010; 19:4176-4188.

44. Heatmaps KRpP. R package version 0.7.7, 2013. https:// cran.r-project.org/web/packages/pheatmap/index.html.

45. Oksanen J, Blanchet FG, Kindt R, Legendre P, Minchin PR, O'Hara RBea. vegan: Community Ecology Package. $\mathrm{R}$ package version 2.0-10, 2013. https://cran.r-project.org/ web/packages/vegan/index.html.

46. Ghirlando R, Felsenfeld G. CTCF: making the right connections. Genes Dev. 2016; 30:881-891. 